EPJ Web of Conferences 63,03005 (2013)

DOI: $10.1051 /$ epjconf/ 20136303005

(C) Owned by the authors, published by EDP Sciences, 2013

\title{
RFQ Reaction Cells for AMS: Developments and Applications
}

\author{
William E. Kieser ${ }^{1, \mathrm{a}}$, Xiaolei Zhao ${ }^{1}$, Albert E. Litherland ${ }^{2}$, John Eliades ${ }^{2}$ and R. Jack Cornett ${ }^{3}$ \\ ${ }^{1}$ Department of Physics \& IsoTrace Lab, University of Ottawa, ON, Canada \\ ${ }^{2}$ Department of Physics \& IsoTrace Lab, University of Toronto, ON, Canada \\ ${ }^{3}$ Department of Earth Sciences \& IsoTrace Lab, University of Ottawa, ON Canada
}

\begin{abstract}
The use of anion-gas interactions in Radiofrequency Quadrupole (RFQ) ion guide reaction cells has been shown to be very effective in the elimination of a number of atomic and molecular isobars which have caused difficulties for Accelerator Mass Spectrometry (AMS) measurements [1,2]. This presentation begins with a review of the early work leading to the use of ion-gas reactions and continues with a discussion the recent measurements of the efficacy of this technique, some of which involve fluoride molecular anions. However, the transformation of the equipment used for these proof-of-principle measurements into a system suitable for routine analysis has required attention to aspects of the ion beam transport and gas handling subsystems. For example, the cross sections of the ion-gas reactions, involving both the analyte ion as well as the isobar, are critically dependent on the ion energy which has to be reduced from the ion source energy, usually between 20 and $80 \mathrm{keV}$, to energies typically in the range of several eV, a task complicated by the energy spread and divergence of beams from AMS sputter sources. With simulations using SIMION 8.1 [3] and tests of promising configurations in a laboratory system, principles for the design of the retarder optics have been developed. These are discussed, along with their planned implementation in a next generation analytical system.
\end{abstract}

\section{Introduction}

Accelerator Mass Spectrometry (AMS) achieves a very high sensitivity for measuring rare atoms and their isotopes partly through its ability to suppress significantly molecular isobars of the atom of interest. The process of removing electrons in either a gas canal or a foil located in the high voltage terminal of the accelerator, in addition to providing the charge changing required for tandem acceleration, facilitates the destruction of molecular isobars through the resulting imbalance of charges on the molecule. The higher energy mass spectrometry then eliminates the molecular fragments and the higher cation energy also facilitates the identification of the lighter elements. In the final ion detector, the large electronic pulses from these cations provide an ion counting system with much lower dark flux of ions compared with lower energy mass spectrometry. For AMS systems using single stage accelerators, a similar charge changing process can take place following the main acceleration stage.

The removal of atomic isobars, in many cases equally important, is more complicated. The earliest AMS work progressed rapidly for cases where the most prolific atomic isobars did not form negative ions. These are to date limited to ${ }^{14} \mathrm{~N}$ in the analysis of ${ }^{14} \mathrm{C}$ [4], ${ }^{26} \mathrm{Mg}$ for ${ }^{26}$ $\mathrm{Al}$ and ${ }^{129} \mathrm{Xe}$ for ${ }^{129} \mathrm{I}$ analysis [5]. Beyond these, aside from very careful sample preparation techniques to exclude the element containing the isobar, the energy available in the accelerated AMS beams permits some additional strategies. The dependence of energy loss on the charge on the nucleus enables the use of foils to either shift the atomic isobar to a lower energy or remove it entirely out of the range of the spectrum of the final detector, as in the case of ${ }^{10} \mathrm{~B}$ for the measurement of ${ }^{10} \mathrm{Be}[6]$. Another energy loss based technique uses a gasfilled magnet. In this case, the ions follow a broader trajectory through the magnetic field that is determined by the average charge state of the ions in the gas. This technique has been used to separate ${ }^{41} \mathrm{~K}$ from ${ }^{41} \mathrm{Ca},{ }^{46} \mathrm{Ti}$ from ${ }^{46} \mathrm{Ca}$ and ${ }^{60} \mathrm{Fe}$ from ${ }^{60} \mathrm{Ni}$ [7]. It is also useful for isobar pairs which can normally be separated in a multi section gas ionization counter, but for which the count rate is too high. Finally, where the atomic number of the isobar is less than that of the analyte and sufficient energy is available, complete stripping can be used, as in the case of the elimination of ${ }^{36} \mathrm{~S}$ in the ${ }^{36} \mathrm{Cl}$ measurements reported in ref [8]. However, all these atomic isobar removal techniques require higher energy, and therefore, more expensive, accelerators to work efficiently.

\footnotetext{
a Corresponding author: wkieser@uottawa.ca
} 
In this presentation, the approach to a method for isobar suppression based on low energy ion-gas collisions will be reviewed and recent developments of this technique will be discussed.

\section{Early Experiments using ion-gas interactions}

For the anion beams used for injection in AMS systems, the possibility of resonant electron exchange between the anion and a gas seemed a possible method for selectively removing atomic isobars from the anion beam. In 1994, Kilius, Litherland and Andrews used low energy $(1 \mathrm{keV})$ beams of a $\mathrm{Cl}, \mathrm{Br}, \mathrm{S}, \mathrm{Cu}, \mathrm{Cu}_{2}$ and $\mathrm{O}$ anions from an 860 type $\mathrm{Cs}$ sputter source to test this possibility in $\mathrm{O}_{2}, \mathrm{~N}_{2}, \mathrm{NO}_{2}$ and $\mathrm{Ar}$ gases at pressures ranging from .02 to $10 \mathrm{~Pa}$ [9]. Their results for $\mathrm{Cl}^{-}$and $\mathrm{S}^{-}$ in $\mathrm{NO}_{2}$ gas are shown in fig 1 . The resulting moderate suppression factor indicated some effect of the ion-gas interaction, but this was obscured by the large degree of multiple scattering of all the ions by the reaction gas. Unfortunately this work was not continued due to the untimely death of Linas Kilius.

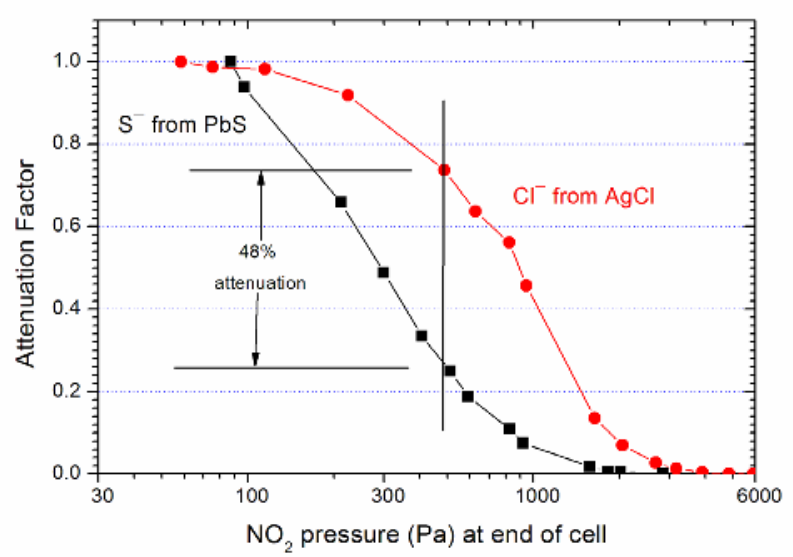

Figure 1. Ion transmission as a function of $\mathrm{NO}_{2}$ gas pressure measured at the end of a $12.7 \mathrm{~mm}$ diameter canal $203 \mathrm{~mm}$ long

Over two decades earlier, studies of electron transfer in $\mathrm{NO}_{2}$ at much lower energies $(\sim 34 \mathrm{meV})$ by Dunkin et al [10] showed that in a mixture of $\mathrm{Cl}_{2}$ and $\mathrm{NO}_{2}$, there was a high probability of transferring an electron from $\mathrm{NO}_{2}^{-}$to $\mathrm{Cl}^{-}$but in the case of $\mathrm{S}$ and $\mathrm{NO}_{2}$, the reaction was more likely to go in the opposite direction, from $\mathrm{S}^{-}$to $\mathrm{NO}_{2}^{-}$. To investigate these reactions under conditions that were closer to those that might be used in an AMS injection line, Doupé, Tomski, Javahery and Cousins [11] carried out an experiment using an atmospheric pressure chemical ionization (APCI) source to generate $\mathrm{Cl}^{-}$and $\mathrm{S}^{-}$ ions which were analyzed in a triple quadrupole system. The second quadrupole was filled with $\mathrm{NO}_{2}$ gas and the third was set to accept any $\mathrm{NO}_{2}{ }^{-}$ions produced. The data from these measurements is reported fully in ref [11] and partly in ref [12] and confirmed the possibility of using such reactions for the suppression of ${ }^{36} \mathrm{~S}$ isobars in the AMS measurement of ${ }^{36} \mathrm{Cl}$. As a result of these explorations, a patent for this general process of isobar separation was obtained [13] and funding for equipment to study these principles directly in an AMS context was acquired.

\section{Measurements with the Isobar Separator}

The apparatus described in the patent [13] and partly implemented (without a cooling cell) on the AMS system at the IsoTrace Laboratory at the University of Toronto (named the Isobar Separator for Anions, or ISA in subsequent publications) is described in references $[1,14]$. The basic layout (shown in Figure 2, ref [12]) includes a standard AMS ion source, an analyzing magnet for mass selection of the beam to be analyzed, a Faraday cup, a constant voltage deceleration section to reduce the beam energy from that emerging form the ion source to several $\mathrm{eV}$, a radiofrequency quadrupole (RFQ) column containing at least one gas cell and a re-acceleration section which restores the ion beam to its original (ion source) energy for injection into the rest of the AMS system. During the course of the exploratory measurements with this system, several RFQ column configurations were tested; the one used for the initial measurement and its operating parameters are described in Figure 1 of ref [1].

In order to demonstrate the isobar suppression principles, which were expected to be of a chemical nature (i.e. element rather than isotope specific) and not strongly mass dependent, macroscopic beams of abundant isotopes (currents of several $\mathrm{nA}$ ) for each element were used. For example, to assess the suppression of ${ }^{36} \mathrm{~S}$ in the analysis of ${ }^{36} \mathrm{Cl}$, beams of ${ }^{32} \mathrm{~S}$, ${ }^{34} \mathrm{~S}$ and ${ }^{35} \mathrm{Cl}$ were used to demonstrate the transmission through the gas cell as a function of gas pressure. With such beams, local Faraday cups could be used for tuning the various control surfaces in the system, although final results were always obtained by passing the beam through the entire AMS system, using the IsoTrace heavy element line [15].

Results for the measurements of the suppression of $\mathrm{S}$ relative to that of $\mathrm{Cl}$ showed a suppression factor of $5 \mathrm{x}$ $10^{-7}$ at a pressure of $\mathrm{NO}_{2}$ gas of $0.8 \mathrm{~Pa}$ (Figure 2, ref [1]). To validate the concept of using abundant isotopes of the elements in question as proxies for the actual analyte and its isobar, these tests were followed by measurements of a ${ }^{36} \mathrm{Cl}$ reference material with ${ }^{36} \mathrm{Cl} /{ }^{35} \mathrm{Cl}=4.16 \times 10^{-11}$ and a dilution series which spanned two orders of magnitude below this level (figure 1, ref [14]). For samples for which no measures were taken to remove sulphur and no prior cleaning of the ion source which had been running $\mathrm{S}$ samples for the elemental tests, the level of ${ }^{36} \mathrm{~S}$ background was calculated to be $3 \times 10^{-14}$. More detailed descriptions of these measurements is provided in the $\mathrm{PhD}$ thesis of J. Eliades [16]

Similar work followed for ${ }^{90} \mathrm{Sr}$ to separate ${ }^{90} \mathrm{Y}$ and ${ }^{90} \mathrm{Zr}$, using the molecular trifluoride anions of ${ }^{88} \mathrm{Sr},{ }^{89} \mathrm{Y}$ and ${ }^{90} \mathrm{Zr}$ and for ${ }^{135} \mathrm{Cs}$ or ${ }^{137} \mathrm{Cs}$ to separate these from ${ }^{135 \text {, }}$ ${ }^{137} \mathrm{Ba}$, using ${ }^{133} \mathrm{Cs}$ and ${ }^{135} \mathrm{Ba}$ difluoride anions [2]. Using the ISA alone, a suppression of yttrium with respect to strontium of $8 \times 10^{-5}$ and a suppression of zirconium with 
respect to strontium of $3 \times 10^{-6}$ were obtained. These ratios are further enhanced by the relative formation probability of the $\mathrm{XF}_{3}{ }^{-}$molecular anion in the ion source (where $\mathrm{X}$ is either $\mathrm{Sr}, \mathrm{Y}$ or $\mathrm{Zr}$ ) of $2 \times 10^{-5}$ for $\mathrm{Y} / \mathrm{Sr}$ and 3 $\mathrm{X} 10^{-5}$ for $\mathrm{Zr} / \mathrm{Sr}$ [2,17]. Similarly, for barium with respect to strontium, the ISA provided a suppression of $2 \times 10^{-5}$, which would be enhanced by a factor of $5 \times 10^{-4}$ for the formation of $\mathrm{CsF}_{2}{ }^{-}$over $\mathrm{BaF}_{2}{ }^{-}$in the ion source. These were preliminary measurements for the demonstration of capability and greater attenuation may be possible with further development.

The analysis of ${ }^{41]} \mathrm{Ca}$ is of interest for biomedical and earth sciences applications [18], but the interference from ${ }^{41} \mathrm{~K}$ usually requires the use of higher energies and thus larger accelerators. Early attempts to use the ISA technique with the molecular fluoride anions $\mathrm{CaF}_{3}^{-}$and $\mathrm{KF}_{3}{ }^{-}$with various reaction gases were only modestly successful $[19,14]$. For example, in $\mathrm{NO}_{2}$, a cell pressure of $1.05 \mathrm{~Pa}$ was required for a $\mathrm{KF}_{3}^{-}$attenuation of $10^{-4}$, but this resulted in an attenuation of the analyte $\mathrm{CaF}_{3}^{-}$by $\sim 8$ $\mathrm{x} 10^{-2}$. A clue for a possible solution to this difficulty was provided in a calculation by Lo and Hopkinson of the structure and binding energies associated with the $\mathrm{KF}_{3}$ anion [20]. They showed that the vertical detachment energy (effectively the binding energy) for the extra electron in this ion is $6.8 \mathrm{eV}$, almost as large as that of $\mathrm{CaF}_{3}^{-}(8.6 \mathrm{eV})$, making $\mathrm{KF}_{3}^{-}$a new type of superhalogen anion. However, they also showed that the energy needed to remove a $\mathrm{F}$ atom from $\mathrm{KF}_{3}{ }^{-}$is only $1.0 \mathrm{eV}$ [21], compared with $3.7 \mathrm{eV}$ for $\mathrm{CaF}_{3}^{-}$. Thus, by maintaining the centre of mass energy of the ions in the reaction cell between 1.0 and $3.7 \mathrm{eV}$ and using collisional dissociation or reactions in gases such as $\mathrm{CH}_{4}$ and $\mathrm{H}_{2}$, the destruction of $\mathrm{KF}_{3}^{-}$could be accomplished with little effect on the $\mathrm{CaF}_{3}{ }^{-}$. With the configuration of the RFQ column used in the earlier measurements, collisions with the reaction gas cooled the ions to below the $1.0 \mathrm{eV}$ threshold energy, resulting in less destruction of the $\mathrm{KF}_{3}{ }^{-}$ than required. Although the gas cell was equipped with gradient electrodes to provide some axial field to maintain the motion of the ions towards the exit of the cell, this field was insufficient to keep the ion energy above the threshold.

One method of maintaining sufficient energy in the ion beam is to use segmented RFQ rods with increasing constant rod offset voltage along the cell [22]. As a simple simulation to test this, the RFQ column was modified to include a second reaction cell coupled directly downstream from the first, with an adjustable constant voltage accelerating gap between them [23]. Rather than use collisional dissociation to remove the $\mathrm{F}$ atom, $\mathrm{CH}_{4}$ was used as a reaction gas, so that the $\mathrm{F}$ atom could be removed by reaction with the available hydrogen atoms attached to the methane. For this gas, a laboratory energy of $10 \mathrm{eV}$ corresponded to a centre of mass energy of $1.4 \mathrm{eV}$. At a cell pressure of $0.5 \mathrm{~Pa}$, an acceleration gap between the cells of $9 \mathrm{~V}$ and a larger than usual voltage on the gradient electrodes in each cell, an attenuation factor of $\mathrm{KF}_{3}{ }^{-} / \mathrm{CaF}_{3}{ }^{-}<10^{-5}$ was achieved [2]. With the implementation of proper segmented rods, it is expected that even more complete attenuation will be achieved.

\section{Entrance lon Optics Design for the Next Generation System}

The need to decelerate the ions from a typical AMS ion source efficiently into an RFQ poses several challenges. As a result of the various processes involved in a sputter source, beams emerge with a considerable divergence, which effectively results from the momentum of the ions in the direction perpendicular to the ion beam. When the ions are decelerated along the direction of the beam axis, their lateral momenta are unchanged, resulting in a significant increase in the divergence angle after deceleration. Secondly, even for a constant ion source acceleration voltage, there is considerable variation in the energy (and hence axial momentum) of the emerging ions and the distribution of this energy shows quite different patterns for atomic in comparison with molecular anions. An example of this distribution for ${ }^{12} \mathrm{C}^{-}$ions and their multimers is given in Figure 3 of ref [24] and for $\mathrm{F}^{-}, \mathrm{HF}_{5}$ and $\mathrm{WF}_{6}^{-}$, in Figure 3 of ref [25]. In both these cases, the atomic anions have a large amplitude high energy tail $(\sim 0.001$ of the peak maximum at $100 \mathrm{eV}$ above the central ion energy), but little low energy tail; the molecular anions show almost no high energy tail, but a smaller but significant low energy tail ( $\sim 10-4$ of the peak maximum at $100 \mathrm{eV}$ below the central ion energy). For a constant voltage deceleration to a central ion energy of 10 $\mathrm{eV}$, most of the low energy tail is lost, but the ions in the high energy tails enter the RFQ column at their original energy offset plus $10 \mathrm{eV}$. Finally, the more the size of the ion beam waist following deceleration is reduced (by adjusting the shape of the deceleration electrodes), the larger the divergence of the on-going beam downstream from that waist.

In order to optimize the deceleration process and to deliver the best beam configuration to the RFQ column, simulations were carried out using Simion 8.1 [3], beginning at the image plane of the magnet upstream from the ISA along to the waist following the deceleration. In general, the results of these simulations indicated that a suitable configuration included a large einzel lens to transport the beam to the deceleration region and a major deceleration gap which reduced the beam energy to $\sim 95 \%$ of its original energy. The waist resulting from the combined effect of the einzel lens and the deceleration gap was found to be optimally located just inside the first section of the RFQ column where the RF field can capture the diverging beam and the constant rod offset voltage complete the deceleration process. A typical configuration diagram and the beam envelope resulting from the projection of 10,000 rays through this system is shown in Fig 2. 


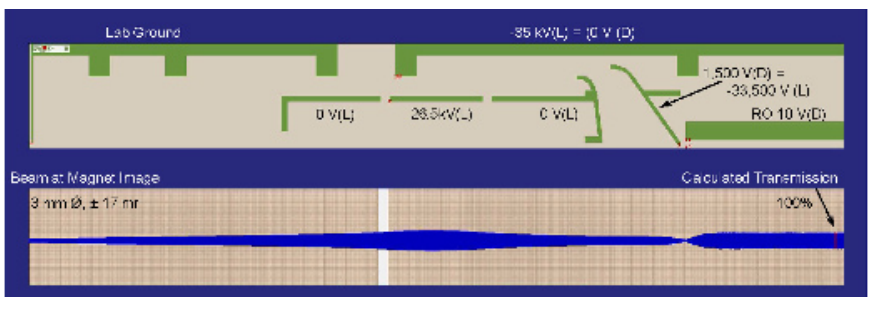

Figure 2. SIMIION 8.1 calculation for the deceleration of an ion beam for a typical lens configuration (upper panel) and the results (lower panel) for a projection of 10,000 rays through this system using the initial conditions and voltages indicated.

\section{Progress on an analytical instrument}

Isobarex Corporation [26], in collaboration with IsoTrace, has been designing a commercial version of the next generation system, which will be tested at IsoTrace as soon as it becomes available. Following from the experience detailed above, the following improvements will be included in this system.

1. The separation of the vacuum chamber into two regions of high vacuum $\left(\sim 10^{-5} \mathrm{~Pa}\right)$ where the large deceleration and acceleration processes take place and a region of lower vacuum $\left(\sim 10^{-3} \mathrm{~Pa}\right)$ surrounding the RFQ column, into which the outflow from the gas cell apertures will vent. This will reduce the probability of anion destruction resulting from gas collisions during the deceleration process. The use of turbopumps rather than the cryopumps used in the present system will permit the use of non-condensable cooling and reaction gases.

2. The use of a fully segmented and modular RFQ column. This will facilitate analytical strategies such as those used in the destruction of $\mathrm{KF}_{3}{ }^{-}$ described above.

3. For a typical AMS system, on which conventional as well as ISA type analyses are planned, the cost of duplicating the ion source and injection magnet presents a considerable economic barrier to the use of the ISA. To combine this functionality into one injection line, the next generation instrument is being designed with an RFQ column which can easily be removed. For normal AMS operations, the large einzel lenses which are part of the deceleration and acceleration regions are reprogrammed to act as a telescopic pair with reduced spherical aberrations to transport the beam from the magnet image plane to the accelerator object point.

A sketch of the ion path is shown in Figure 3.

\section{Summary and Conclusions}

The use of ion-gas interactions in the AMS analyses of ${ }^{36} \mathrm{Cl},{ }^{41} \mathrm{Ca},{ }^{90} \mathrm{Sr}$ and ${ }^{135,}{ }^{137} \mathrm{Cs}$ is now a convincing strategy for the suppression of the atomic isobars that complicate these measurements. While the use of these techniques will improve the sensitivity and detection limit for any AMS system on which it is used, it will be particularly

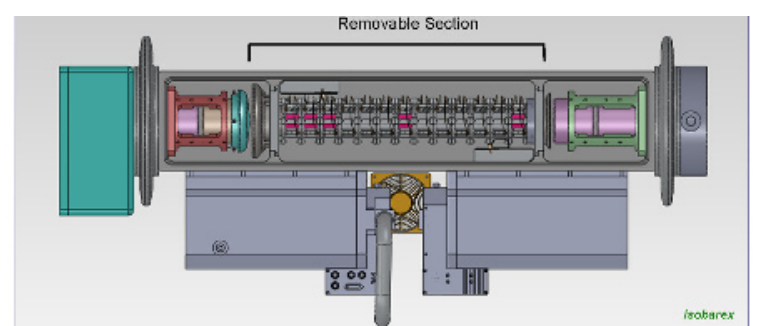

Figure 3. Drawing of an Isobarex ISA showing the removable elements of the ion path.

effective for the smaller and less expensive systems that are now being used. The extension of this technique to other rare species may simply be a matter of determining the appropriate procedures, cooling and reaction gases and the correct instrument operating parameters. However, the case of ${ }^{41} \mathrm{CaF}_{3}^{-}$and the structure of the isobar ${ }^{41} \mathrm{KF}_{3}{ }^{-}$described above and in ref. [23] indicates the need for much further research into the physical chemistry of such superhalogen anion - and may lead to greater communication between AMS developers and theoretical chemists. Finally, the existence of an adaptable commercial instrument will make this technology more available to a larger group of researchers and thus increase our knowledge of anion-gas interactions.

\section{Acknowledgements}

The financial support from the Idea to Innovation, Major Resources Support and, for WEK and AEL, the Discovery Grant programs of the Canadian Natural Sciences and Engineering Research Council, as well as more recently, from the CRTI program of the Centre for Security Science, Canada and the University of Ottawa is all gratefully acknowledged. This work has benefitted greatly from the early seminal work by Ilia Tomski and Jonathan Doupé, the initial measurements, the continuing support of Reza Javahery and Lisa Cousins of Ionics Mass Spectrometry Group and from the on-going discussions with Jean-François Alary of Isobarex Corporation.

\section{References}

1. J. Eliades, A.E. Litherland, W. E. Kieser, L. Cousins, S. J. Ye, and X-L. Zhao, Nuclear Instruments and Methods B 268, 839 (2010)

2. J. Eliades, X.-L. Zhao, A.E. Litherland, and W.E. Kieser, Nuclear Instruments and Methods B 294, 361 (2013)

3. SIMION 8.1, Scientific Instrument Services, Inc., http://simion.com/

4. K.H, Purser, R.B. Liebert, A.E. Litherland, R.P. Beukens, H.E. Gove, C.L.Bennet, M.R. Clover and W.E. Sondheim, Revue de Physique Appliquée 12, 1487 (1977) 
5. A.E. Litherland, Ann. Rev. Nuclear and Particle Science 30, 437 (1980)

6. G.M. Raisbeck, F. Yiou, D. Bourles, E. Brown, D. Deboffle, P. Jouhanneau, J. Lestringuez and Z.Q. Zhou, Nuclear Instruments and Methods B 92, 43 (1994)

7. M. Paul, Nuclear Instruments and Methods B 52, 315 (1990)

8. A. Galindo-Uribarri, J.R. Beene, M. Danchev, J. Doupé, B. Fuentes, J. Gomez del Campo, P.A. Hausladen, R.C. Juras, J.F. Liang, A.E. Litherland, Y. Liu, M.J. Meigs, G.D. Mills, P.E. Mueller, E. Padilla-Rodal, J. Pavan, J.W. Sinclair and D.W. Stracener, Nuclear Instruments and Methods B 259, 123 (2007)

9. L.R. Kilius, A.E. Litherland and H.R. Andrews, IsoTrace Laboratory Annual Report 42 (1994)

10. D.B. Dunkin, F.C. Fehsenfeld and E.E. Ferguson, Chemical Physics Letters 15257 (1972).

11. J.P. Doupé, PhD Thesis, University of Toronto (2004)

12. A.E. Litherland, I. Tomski, X.L. Zhao, L. Cousins, J.P. Doupé, G. Javahery and W.E. Kieser, Nuclear Instruments \& Methods in Physics Research B 259230 (2007)

13. A.E. Litherland, J. Doupé, W.E. Kieser, X-L Zhao, G. Javaheri, L. Cousins and I. Tomski, A. U.S. Patent No 7,439,498 (2008)

14. W.E. Kieser, J. Eliades, A. E. Litherland, X-L. Zhao, L. Cousins and S. J. Ye, Radiocarbon 52, 236 (2010)

15. L.R. Kilius, N. Baba, M.A. Garwan, A.E. Litherland, M.-J. Nadeau, J.C. Rucklidge, G.C. Wilson and X.-L. Zhao, Nuclear Instruments \& Methods in Physics Research B 52357 (1990).

16. J. Eliades, $\mathrm{PhD}$ theseis, University of Toronto 2012

17. X-L. Zhao, A.E. Litherland, J. Eliades, W.E. Kieser and Q. Liu, Nuclear Instruments and Methods B 268, 807 (2010)

18. S.P.H.T. Freeman, J.C. King, N.E. Vieira, L.R. Woodhouse and A.L. Yergey, Nuclear Instruments and Methods B 123, 266 (1997)

19. X-L. Zhao, J. Eliades, Q. Liu, W.E. Kieser, A.E. Litherland, S. Ye and L. Cousins, Nuclear Instruments and Methods B 268, 816 (2010)

20. S. Lo and A.C. Hopkinson, Computational and Theoretical Chemistry 97312 (2011)

21. A.C. Hopkinson, private communication

22. G. Javahery and B Thompson, Journal of the American Society for Mass Spectrometry 8, 697 (1997)

23. X-L. Zhao, A.E. Litherland, J. Eliades, Y-C. Fu, and W.E. Kieser, Radiocarbon in press

24. H. Gnaser, Nuclear Instruments and Methods B 26637 (2008)

25. H. Gnaser and R. Golser, Surface and Interface Analysis 4337 (2010)

26. Isobarex Corporation, 32 Nixon Road. Unit \#1. Bolton ON L7E1W2, Canada www.isobarex.ca 